HIBTOLOGIOAL NOTES ON A OABE OF TABES WITH OPHTHAL. moplegia externa. By Dr. Betay Lerir.

\title{
The Spinal Cord.
}

Seotions taken indiscriminately from any region of the cord exhibit very advanced degenerative changes invariably limited to the posterior column. The lesion, which is of the nature of grey degeneration, is fairly symmetrical in its hemispherio distribution throughout the different regions of the cord; but there is evidence in the distorted marginal contour of these columns in the lumbar cord (due to sclerous shrinking and contraction) that the lower realms have been earlier implicated, as they exhibit a later stage of sclerosis that what obtains in the higher divisions of the cord. To the naked eye all transverse sections when stained display the posterior columns mapped out with extreme distinctness by the far greater depth of tint which they attain in contrast with that of the antero-lateral columns. Aniline staining in this respect may be most favourably compared with the carmine sections, and exhibit with even greater distinctness this morbid coloration due to the preponderance of the connective elements. Similar features are observed under the low powers of the microscope, and the nature of the change indicated by the great increase of intermedullary connective which, invading irregularly the different portions of the tissue, separate whole fascicles of unaffected medullated tubes from one another by the intervening deepstained sclerous tissue. The latter consists not alone of intermedullary fibrillated connective, but of numerous multi-candate cells, which differ only in number, size, and readiness of staining from the normal cells of the medullary septe supporting the vascular network of the cord. These morbid elements usually measured $.027 \mathrm{~mm}$. $\times .013 \mathrm{~mm}$., were extremely irregular in size and contour, threw out numerous delicate fibrous prolongations, and were generally associated with large, dilated, and tortuous blood vessels, the walls of which were covered by proliferating nuclei. Throughout the whole commissural and radicular zones of the posterior columns, these 
vessels appeared both more numerous and more dilated than wes the case in the normal cord used for comparison with the morbid specimens.

The spider-like connective cells were far more numerons in the post-commissural than in the posterior radicular zones, and were very scanty or wholly absent in the columns of Goll, where the fine fibrillated intervening connective predominated as the morbid element. In all the minute medullated tubes, the wasting of the medullary sheath was revealed at a glance by the great disproportionate size of the exis-cylinder, whilst in those of wider calibre measnrements gave for large numbers an axis-cylinder of $.006 \mathrm{~mm}$., or $009 \mathrm{~mm}$. for medullated tubes of $011 \mathrm{~mm}$. - a very appreciable degree of wasting. In the more advanced foci of sclerous change the medullary sheath was entirely absent, and remains of axis-cylinders dntted the embracing zones of connective. The regional distribution of the lesion exhibited the following characters from above downwards.

a. Cervical enlargement.-The most extreme sclerous change was observed in the medullated zone bordering upon the posterior commissure (post-commissural zone), which appeared to have been a focus of the most intense degenerative alteration. To the finely punctuated appearance given by the normal axiscylinders is here superadded a mosaic of deeper and fainter stained areas corresponding to sites more or less sclerosed, and with this is conjoined a coarse dotting of the field due to the great increase in numbers of deeply-stained connective cells, and the divided orifices of numerous coarse blood-vessels. Immediately behind the commissure the medullated fibres are almost universally reduced in diameter, and although a few tubuli are seen, which attain the average large diameter of this zone ( $014 \mathrm{~mm}$.), and a few exceptional ones even rival those of the columns of Turck ( $027 \mathrm{~mm}$.), still the vast proportion are extremely reduced, whilst extensive sclerosed tracts embrace the remains of axis-cylinders or the minutest medullated tubes. It was in this region, as before stated, that the connective cell and distended vessel predominated. Next to this post-commissural zone, the radicular zones are the parts most gravely implicated-the anterior series of radicular fibres 
suffering more severely than those behind them, the integrity of which may be clearly traced throughout an exterisive course. A somewhat large islet of nearly healthy tissue bordered the more anterior segment of these radicular zones (cols. of Burdach), but the posterior segments of the same were sclerosed throughout. The median columns (cols. of Goll) were degenerated to an equal degres throughout the whole or greater part of their extent.

B. Dorsal region.-Here, as in the upper realms, the most intense change was concentrated upon the large medullated elements bordering upon the posterior commissure, whence the sclerous tissue fringed the columns of Burdach on either side, implicating to a very serious extent the posterior radicular fibres. Far more universally involved and degenerated than in the cervical cord, these radicular fibres were often completely destroyed, the islets' of healthy tissue being very few, and surrounded on all sides by large quantities of inter-mednllary connective, branched cells, and dilated, tortuous vessels. The sclerous change has also invaded in this region of the cord the posterior cornua as far as the emergence of the posterior roots, the change being somewhat more marked on one side than the other, a statement which also applies to the posterior radicular fibres. A very limited patch of degeneration is found in the median district, which, on either side of the so-called posterior fissure, is otherwise wholly unaffected by the lesion.

$\gamma$. Lumbar enlargement.-Bordering the posterior fissure on either side are two riband-like bands of deeply-stained sclerosed tissue. By subsequent contraction of this tissue the two lateral halves of the posterior columns have been almost completely rent asunder, being connected only by a slight bridge of degenerated tissue near the posterior margin of the cord. These retracted sides enclose an elliptic space betwixt them of some size, so that the area of the posterior columns is greatly reduced in extent. A similar dense sclerous tissue follows out the posterior border of the cord, extending to within a very short distance of the posterior roots. Apart, however, from this morbid fringe along the raphe and borders of the posterior columns, we also find advanced degenerative changes in the post-commissural zone, similar in site and in character to what 
was described in higher régions, stopping short at the radicular zones, the fibres of which are less implicated than in any other region of the cord. In the lowest part of the lumbar enlargement, on the right side, a peculinr cyst-like cavity, $1.5 \mathrm{~mm}$. deep by $.75 \mathrm{~mm}$. wide, exists in the posterior cormu. It has condensed sclerosed margins, and occupies the site of a large proportion of the vertical fibres of this cornu, which ascend in front of the substantia gelatinose. The fibres (horizontal) of the liatter arch round it on either side, but not completely surrounding it, and appear compressed together by the former contents of the cavity which have now escaped. A few of the vertical fasciculi still remain intact.

Antero-lateral columns.-A careful examination of these columns in the cervical, dorsal, and lumbar regions was made, and the results enable me to state positively that the (a) direct cerebellar tracts; $(b)$ the direct and crossed pyramidal tracts, and (o) Gowers' tracts were free from any appreciable lesion, the histological elements being well displayed and perfectly normal.

Anterior cornua.-A similar statement may be made for the anterior horns of grey matter. The nouroglia had a perfectly normal espect; the small escending fasciculi of minute mednllated fibres were well developed; the nerve-cells were plump, slightly pigmented, and exhibited neither quantitative nor qualitative change: Their marginal fibres for the motor rcots were beautifully shown in all sections and free from any morbid change, nor in any region was the slightest tendenoy exhibited to extension of the lesion from the posterior cornue.

To summarise the changes observed:-

a. Most advanced sclerous change in the post-commissural zones throughout all the regions of the spinal cord alike.

b. Extension along the posterior radicular zones of a minor degree of intensity, and implicating the radicular fibres causing their destruction to a slight extent in the cervical, to a still less extent in the lumbar, but to a very grave extent in the dorsal region.

c. The columns of Goll through the cervical region were almost completely sclerosed, but the degree of degenerative change was less than in the above-mentioned regions. 
d. Median segment of dorsal posterior columns scarcely affected.

e. Median raphe and posterior border of the same columns in the lumbar cord sclerosed and distorted.

$f$. Small cyst-like cavity in gelatinous substance of posterior cornua in the lumbar region on the right side.

g. Implication of the posterior cornua by the same grey dogeneration, but to a slight degree in both cervical and dorsal regions, extending usually to point of emergence of posterior roots.

$h$. In the sites of most advanced sclerosis (post-commissural and post-radicular zones) the size and number of the dilated bloodvessels formed a notable feature under a low objective.

i. Numerous amyloid bodies appear scattered throughout the posterior columns of most of the regions of the cord.

The pons and medulla.-Sections were carried through the nuclei of origin of the various bulbar nerves. Of those found along the lower half of the floor of the fourth ventricle-the accessory, hypoglossal, vagus - and glosso-pharyngeal-the nuclei as well as the emergent or radicular fibres were found well displayed and perfectly intact. No diseased tract of tissue was anywhere apparent in the transverse sectional areas of this district, and the restiform columna and olivary nuclei and fibres were throughout free from lesion. Upon reaching the higher level of the origin of the sixth nerve, undoubted evidence was obtained of a morbid condition of this tract. The lésion was obviously a vascular one, and had secondarily implicated the emergent fibres from the abducens-facialis nucleus-the root fibres of the sixth pair. The nucleus, where it lies in front of the genu of the facial nerve, was represented by mere remnants of degenerated cells, and a punctated aspect of tissue from the resulting débris. The fibres of origin of the abducens, usually so clearly defined within the field embraced by the band of facial roots, were represented merely by an occasional narrow streak of minute and degenerated fasciculi teking their usual curved course, but lost to view after a short distance. Along the direction pursued by these diseased fasciculi were several large swollen and tortuous blood-vessels perfectly occluded by därk clots, their coats extensively diseased and frequently crowded by heaps of red blood cor- 


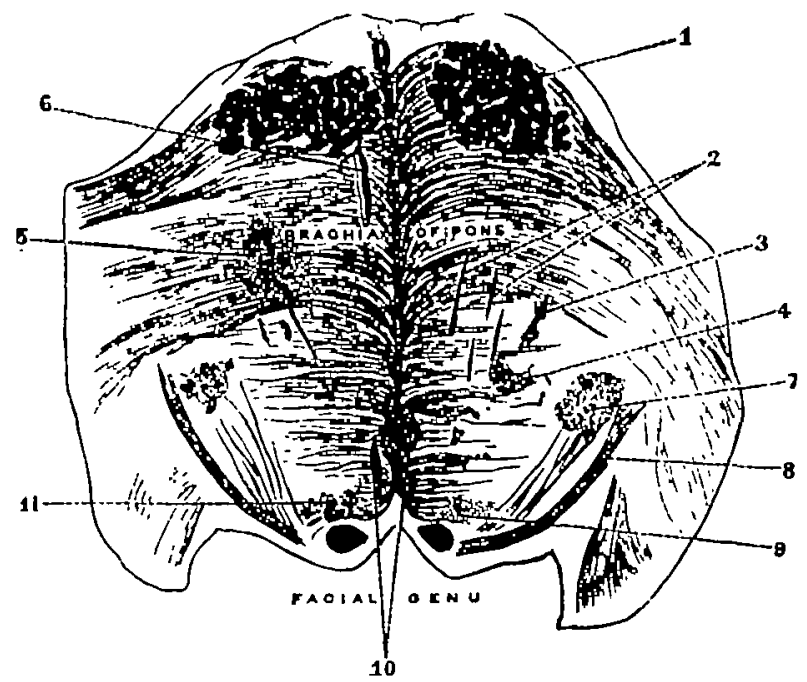

1. Vertical fasctedl from crura.

2. Remeins of root of aisth nerve.

3. Diseased and plusged vessels corored with blood corpuelea.

4. Miliary extravantion.

6. Lergo focus of extrevaution. [plagged.

6. Blood-vesiel in adranced itate of disease,
1. Fonlerior nucleas and arceirding routs of tho facial.

8. Fadnl nerreter

9, 11. Abduocno fecilily deginerated on both Idea.

10. Dheased and plugged rersel.

puscles. Along the course of the same fibres of the sixth pair are several miliary apoplexies, which have probably induced the degenerative changes here observed. Apart however from these foci of extravasation, we find also innumerable red corpuscles crowding certain parts of the field on either side of the median raphe; in fact the region of the abducens has been the seat of miliary apoplexies extensively induced by plugging of the nutrient vessels of this realm. In the rough sketch appended, I have endeavoured to localise a few of these diseased vessels and extravasations in a section taken at this level.

Level of Lemniscus.-Those vertical medullated fasciculi which linit the tegmentum in front, and are embraced by the most posterior fibres of the brachia of the pons, exhibit a notable increase of their connective elements. These fibres, associated with those from the fillet of the testes as far as the median raphe, have interspersed amongst them numerous islets 
of sclerosed tissue, in which the remains of degenerated medullary tubes are well seen. The whole sectional area of the fillet lying in front of the tegmentum was thus involved, but the tracts of grey degeneration were not extensive, being scattered apart, not in circular patches like distinct focal lesions.

The upper realms of the pons and medulla had unfortunately incurred such damage during the process of preservation as to preclude an exhaustive examination of the nuclei of origin of the remaining oculo-motor nerves.

In the case of A. B. we have all the symptoms of tabes dorsalis. There are pains sharp and sudden in character, an ataxic gait, absence of knee phenomenon, loss of pupillary reflex, loss of muscular sense, some cutaneous anæsthesia, gastric crises. But beyond these symptoms there is another of rarity and interest. The symmetrical immobility of the eyeballs accompanied by more or less ptosis of the lids is a condition which was first described by Graefe under the name of ophthalmoplegia progressiva. An account of it is contained in the 'Lehrbuch der functionellen Nervenkrankheiten,' by Eulenburg (Berlin, 1871). I can remember that, nine or ten years ago, shortly after reading that account, I saw an example of the disease in St. Bartholomew's Hospital. The patient was a female, under the care, I think, of Dr. Andrew, and she had this symmetrical immobility of the eyeballs typically marked. The disease has more recently received a most cearching investigation at the hands of $\mathrm{Mr}$. Hutchinson, who has applied to it the term ophthalmoplegia externa," in contradistinction to that of ophthalmoplegia interna, which he had given in a previous paper, to a state of immobility of the pupil, in which the internal muscles of the eye (the iris and ciliary muscles) are together involved in paralysis. He, however, explains that the former term must be understood as including very frequently, if not usually, the immobility of pupil.

Mr. Hutchinson's description of the condition is so graphic that'I cannot possibly do better than quote it here:- " Drooping of the eyelids," he writes, "so as to give to the face a half- 
asleep expression, is usually the first symptom, and it is soon accompanied by weakness of all the muscles attached to the eyeball, so that the movements of the latter become much restricted, or even wholly lost. The condition is usually bilateral, though it is not always exactly the same in degree on the two sides. Its symmetry probably denotes that it is of central origin. It by no means always happons that all the ocular muscles -are alike affected, or. that they are attacked simultaneously, still it is a very marked feature of the malady that the muscles fail "in groups and not singly." Seventeen cases of the kind are described in the paper. In ten of these it seemed certain that syphilis was the cause; in eight acquired, and in two inherited. Of the remaining seven Mr. Hutchinson remarks, "it may be said that a reasonable suspicion of syphilis might be entertained in several." In one case an examination of the brain after death was made by Dr. Gowers, who found that degenerative changes precisely similar to those seen in progressive muscular atrophy had implicated the origins of the third, fourth, and sixth nerves as well as, in that particular instance, the optic nerves also. The large nervecells had disappeared from the nuclei of origin of these nerves.

Mr. Hutchinson remarks that we have in such cases a very close parallel to the so-called bulbar paralysis-the labio-glossolaryngeal paralysis of Duchenne-and he thinks it may be plausibly conjectured that the initial lesion is inflammation of the nuclei of the affected nerves, which, in a slowly serpiginous manner, creeps from place to place along certain definite anatomical paths. In my female patient the history of syphilis was clear and certain. On the other hand, there is no evidence that X. Y. hod ever suffered from the infection. The condition of his aorta is a suspicious circumstance, but the history of rheumatic fever prevents us from laying stress upon the condition as indicative of constitutional syphilis. In any case, however, the proximate cause of the lesions of nuclei must be sought for in an extensively diffused disease of the vascular system, of which there is evidence in the dilatation of the aorta, the rigidity and tortuosity of the brachiad arteries, no less than in the microscopical changes in the intrabulbar arterioles with the resulting miliary hæmorrhages. 
In A. B. it will be observed there is a symptom which (as it happens) is not described as occurring in any of those related by $\mathrm{Mr}$. Hutchinson. I refer to the atrophy of muscles about the back and shoulders. This association, however, evidently tends to support the view that the lesion of the nuclei of origin of the cranial nerves is prebably of the same nature as that which determines progressive muscular atrophy. In Charcot's lateral amyotrophic sclerosis there is atrophy. of the large motor cells in the anterior cornus of the cord. The disease tends, as we know, to travel upwards, and in time spreads to the medulla oblongata. Attacking there the nuclei of the hypoglossal, facial, and trigeminal (its motor portion), it canses death by involving also the nucleus of the vagus. Now, it seems probable that, were it not for the fatality necessarily attaching to the destruction of this nuclens, the disense would also, in those cases, be likely to invade the nuclei of origin of. the oculo-motor nerves, and so bring about the condition of the eyes which is to be seen in this girl. Death, in fact, stops the progress of the disease before it invades the region devoted to the innervation of the external and internal muscles of the eye.

The lesion of nerve nuclei would appear, in the case of A. B., to be a more or less continuous one, from the intracranial centres for the higher cranial nerves to the anterior cornua of the cord. On the right side the origin of the facial nerve is involved, though not apparently on the left. The quivering of the tongue shows that the nucleus of the hypoglossal is probably not intact.

In the atrophy of the sternomastoids and left trapezius there is evidence of invasion of the nuclei of the accessory.

I have but little doubt that the gastric crises (as I have some time ago suggested ${ }^{1}$ ) depend upon irritation of the nucleus of the vagus by sclerosis. This opinion has since received support by the result of an observation of Pierret, who found in a case of tabes, with characteristic gastric crises, sclerosis of the fasciculus gracilis in immediate relation with the nucleus of the vagus. The fibres of the vagus and accessory are in such close proximity at their point of origin that chronic inflammation of

1 Pathological Bociety, Febraerr. 1880. 
the immediately adjacent tissues may be expected to cause both sensory and motor disturbance-pain in the stomach and vomiting.

The symptom of intense hunger; of which this girl complained, is one that I have on several occasions observed in connection with the occurrence of gastric crises in tabes. It was marked in a case of tabes with gastric crises and joint affection under the care of my friend, Mr. Herbert Page.

It is present also in the case of a man, suffering from tabes with gastric crises, who is now under my care in the hospital.

Only a few woeks ago, whilst looking throngh 'A System of Clinical Medicine,' by Dr. Graxes, published in 1843, I chanced upon a description of gastric crises which is so graphic that I cannot refrain from quoting here a rather full abstract of the case narrated. Viewed by the light of our increased knowledge, the case is evidently one of tabes dorsalis, but it occurred long before this disease was differentiated from other forms of paraplegia. The antopsy, which included examination of the brain, spinal cord and nerves, besides the abdominal viscera, naturally failed to throw light apon the cause of the disease; for in those days the methods of hardening preparations of nerve substance, the initiation of which we owe to Lockhart Clarke, had not boen devised, and the examination, as far as I can judge, was only made with the naked eye. Dr. Greves appears, however, to have been curiously near the true solution in a remark which he makes. "Are we to attribute," he writes, "this diseased condition of the stomach and bowels, which, from the remarkable periodicity of its occurrence, was evidently functional, to irritation, congestion, or inflammation of the brain or spinal marrow? From the date we are in possession of it appears that this question must be answered in the negative." He speaks of the case as a very remarkable one, which had made a great impression upon him, and, in his view, well worthy of the attention of the pathological inquirer.

The patient, Mr. B., aged 23, was exceedingly strong, and passionately fond of hunting, fishing and shooting. These habits, however, he laid aside aiter the occurrence of the first attack of his illness, which happened in 1829. From that time 
his bowels, previously sluggish, became inclined to looseness, which always increased before the appearance of one of the attacks, accompanied by griping, nausea and inclination to vomit. Each attack was generally preceded by a copious secretion of insipid watery fluid in the mouth, and then the characteristic symptoms of his disease commenced. These consisted in obstinate and protracted nausea and romiting. He first threw up whatever happened to be on his stomach at the time, and afterwards everything he swallowed, whether solid or liquid, and the quantity ejected in the course of a day varied from three to four quarts of fluid. He complained also of pain, referred to the stomach or lower part' of the chest, which continued throughout the attack, being most acute at its commencement; for the last year this sensation had passed into a feeling of painful constriction, which he described as a "contracted feeling of his inside," and compared' it to something like the effects of a cord drawn tightly, so as to compress or atrangulate his body exactly along the outline occupied by the insertions of the diaphragm. During the prevalence of the attack he had profuse perspirations, particularly towards the termination of each paroxysm. The duration of the first attack did not exceed four or five days, after which he became quite well, and continued so for six or seven months, when his symptoms suddenly returned. He began to reject everything from his stomach as before, but in the course of a few days the vomiting disappeared, and for a considerable interval he had no return of his complaint. In the year 1830 he had three attacks of a similar description; from these he recovered also completely, and without remarking any diminution of power in his lower extremities. In 1831, however, the disease began to assume a more serious aspect; the paroxysms became much increased in severity, lasted longer and recurred at shorter intervals. For one of these attacks he took mercury, and was salivated. In 1832 his symptoms became still more violent, and the duration of the paroxysms more protracted. He had one in March, a second in May and a third in June, each of which was accompanied by some numbness and loss of power in the lower extremities ; this, however, was slight, and disappeared altogether as the vomiting subsided. About this time 
he noticed that his urine was scanty, and deposited more sediment than nsual. He also complained of being very apt to catch cold whenever he got out of bed, and stated that he suffered occasionally from severe twitches and pains in his legs, thighs, arms and other parts of his body, which were generally succeoded and carried off by profuse perspirations.

In August, 1832, he had a violent attack, which lasted nearly a month. The romiting was incessant, continuing night and day, and he suffered severely from the feeling of painful constriction already described. On getting up after this attack his legs suddenly failed him, and he dropped down on the floor quite powerless. The paralysis did not now disappear during the intervals, although it grew somewhat better after each flt of romiting had ceased; indeed he used to improve in his walking after the paroxysm had entirely disappeared, and, aided by two sticks, supported himself so as to give some hopes of a recovery, until a recurrence of his attack reduced him again to a state of almost total paraplegia. His legs now began to waste sensibly, and he noticed that they had lost their foeling and were remarkably cold. He also complained of severe twitches of pain in various parts of his body, accompanied by profuse night sweats, and turbid scanty urine.

For some months before his death he was completely paraplegic, and continued to be attacked with violent fits of vomiting. The vomiting went on night and day, and he was unable to retain the mildest and most soothing substances for a moment on his stomach. Everything was tried to alliny the irritability of the stomach, but in vain. After continuing to resist obstinately every form of treatment for five or six days and nights the vomiting would suddenly cerse, the gentleman would exclaim, "Now I am well," and he could then eat with perfect impunity substances of an indigestible character. The transition from a state of deadly nausea and obstinate retching to a sharp feeling of hunger used to occur quite suddenly. One hour he was a miserable object, rejecting everything, and suffering the most painful constrictions across the epigastrium, the next found him eating with a voracious 
appotite whatever he could lay hold of, and digesting everything with apparent facility.

Here again we have the symptom of hunger described very graphically. I would submit that it tends to corroborate the view that the seat of lesion occasioning gastric crises is in the neighbourhood of the pneumogastric nucleus.

It may be asked why, if we have to do here with irritation of this nucleus, we should not, in cases of tabes with gastric crises, also meet with symptoms referable to the heart and to the respiratory apparatus. We do, in fact, sometimes meet with laryngeal crises, and rapidity of pulse is of no unfrequent occurrence in the course of tabes dorsalis. It does not seem reasonable, however, to expect that in these circumstances we should necessarily find evidence of impairment in all the functions subserved by the nerve.

It cannot be doubted, I think, that there is a very elaborate differentiation of functions of the nerve-cells constituting a nucleus. In a paper of great interest and importance ${ }^{2}$ Dr. Felix Semon calls attention to the proclivity of the abductor fibres of the recurrent laryngeal nerve to become affected sooner than the adductor fibres, or even exclusively, in cases of undoubted central as well as peripheral injury or disase of the roots or trunks of the pneumo-gastric, spinal accessory, os recurrent nerves.

The differing amount of paralysis in the muscles of this girl's eyeballs and lids is also indicative of differentiation of function in various parts of the nuclei of the oculo-motor nerves. ${ }^{3}$

I do not think, therefore, that we shall see anything remarkable in irritation of the nucleus of the vagus in a particular case being followed by symptoms entirely referable to the stomach or intestines. Whilst upon this point I would remark that, in certain cases of tabes where the more typical form of gastric crises has not been marked, there has been,

1 Several cases of typical gastric orises will be found degcribed in my paper on " Certain Little Reconnised Phases of Tabes Dorsalis," Transactions of the Intornational Med. Congross, 1881.

- Boo 'Archives of Laryngology,' vol. ii. No. 3, Jaly, 1881.

- Dr. Sturge has publiahed some interesting remarks on this subjoct. "Tro cases of simultaneous paralysis of both third nerves."- Ophthalmological Society's Transactions' vol. i.

VOL. $\mathbf{7}$. 
nevertheless, a peculiar tendeney not so much to diarrhoon ns to abnormally frequent action of the bowels, the evacuations not being necessarily loose. I think it probable that this symptom, like the pain in the stomach and romiting, may prove to depend upon irritation of the nucleus in question, for it must be remembered that the peristaltic action of the small intestine is largely influenced by nerrous impulses prssing along the splanchnic and vagus nerves. According to Pflügor, whilst stimulation of the splanchnic nerves tends to check the peristaltic movements, that of the vagus is calculated to excito them. ${ }^{2}$

In reference to this question of the dependence of gastric crises (of the two kinds which I have mentioned) upon sclerosis in the neighbourhood of the nucleus of the pneumo-gastric, I would call attention to a point which, although of purely negative character, seems to me to be of considerable importance.

The man X. Y. did not suffer at all from gastric crises. It will be observed that $\mathrm{Dr}$. Bevan Lewis in this case found the nuclei of the vagus amongst other nerres well displayed and perfectly intact, and he adds, " no diseased tract of tissue was anywhere apparent in the transverse sectional areas of this district." The importance of this observation will be especially evident when it is remembered that the funiculus cuneatus and gracilis, which closely adjoin the sensory portion of this nncleus, represent the continuation upwards to the cerebellum through the medulla oblongata of the posterior columns of the spinal cord.

Let me note here an important difference in these two cases. The muscular-atrophy which affects in a marked manner the trunk and extremities of the patient A. B., was entirely absent in the case of the man X. Y. The microscopical findings corresponded with the clinical history. (1 ought here to say, perhaps, that I purposely kept Dr. Bevan Lewis in ignorance of the symptoms observed during life, in order that his observations might be quite unprejudiced.) Dr. Lewis reports that the anterior cornun of the spinal cord were entirely free from any morbid change.

I 'A Text-book of Plıysiology." By M. Foster. 3rd clition, p. 207. 
It is interesting to observe that Mr. Hutchinson found " in six of his cases the lower extremities more or less weak, and a condition approsching more or less closely to locomotor ataxy" and he makes the important remark, that "there can be no doubt that ophthalmoplegia externa is sometimes a part of the general malady known as progressive locomotor ataxia, especially when that disease is due to syphilis." The two instances which I have described are examples, at all events, of the first described association. Both patients presented the most characteristic symptoms of tabes dorsalis. In each there were "lightning" or "electric" pains, absence of knee phenomenon and ataxy. No other signs need mention in the presence of these. 